\title{
Estimates on the Vorticity of Solutions to the Navier-Stokes Equations *
}

\author{
Vladimir Scheffer \\ Department of Mathematics, Rutgers University, New Brunswick, NJ 08903, USA
}

\begin{abstract}
We estimate the vorticity of the flow of an incompressible, viscous, three dimensional fluid near the boundary of its container. We obtain a bound that is valid outside a small subset of space-time with special properties.
\end{abstract}

\section{Introduction}

It is the basis of the Prandtl boundary layer theory that vorticity is introduced into solutions of the Navier-Stokes equations through a boundary layer. Therefore, it is important to obtain estimates of the size of the vorticity close to the boundary. The theorem below yields the following type of estimate: We fix a small positive number $\tau$ and examine points $(x, t)$ in space-time, where $x$ lies at a distance $\tau$ from the boundary. We also assume that the time elapsed since the beginning of the flow is at least $\tau^{2}$. Then the size of the vorticity at $(x, t)$ is at most $O\left(\tau^{-2}\right)$ unless $(x, t)$ happens to lie in a certain set. This set is the union of cylinders of size $\tau$. The number of different cylinders is at most $O\left(\tau^{-5 / 3}\right)$. Since the cylinders are subsets of space-time, their union is a small set. However, the important point is not the measure of this set. The interesting thing is the clustering of this set into lumps of size $\tau$. Outside of these lumps we have uniform estimates on the vorticity.

The proof of the main theorem in [1] involved the construction of a similar set. There the set was the union of finitely many cylinders $A_{i}$ where $\sum_{i}$ (diameter of $\left.A_{i}\right)^{5 / 3}$ was bounded by a constant that depended only on the initial kinetic energy. In addition, the maximum of the diameters of the $A_{i}$ could be made arbitrarily small. The theorem below is an improvement on this.

One can go further and state that the vorticity is continuous at the points where we can estimate its size. This is a consequence of the local boundedness

* This research was supported in part by the National Science Foundation Grant MCS-7903361 
of the velocity vector field at these points (this is brought out in the proof) and of the argument that led to partial continuity of the vorticity in [1].

In this paper the vorticity is the curl in the distribution sense of the velocity vector field. It is not difficult to show that it is actually a classical curl at the points at which it is estimated. In other words, the spatial partial derivatives of the velocity vector field exist in the classical sense at each such point.

Theorem. Let $U$ be an open bounded subset of $R^{3}$ such that the boundary of $U$ has Lebesgue measure zero. Suppose that $w^{0}: R^{3} \rightarrow R^{3}$ is an $L^{2}$ function such that $w^{0}(x)=0$ for $x \notin U$ and $\operatorname{div}\left(w^{0}\right)=0$. Then there exists a weak solution $u: U$ $\times(0, \infty) \rightarrow R^{3}$ to the Navier-Stokes equations of incompressible fluid flow in $U$ with adherence at the boundary of $U$, initial condition $w^{0}$, viscosity $=1$, and the following property: If $0<\tau<1$ and

$$
T=\left\{(x, t) \in R^{3} \times R: t \geqq \tau^{2} \text { and distance }\left(x, R^{3} \sim U\right) \geqq \tau\right\},
$$

then there exist $\left(x_{1}, t_{1}\right),\left(x_{2}, t_{2}\right), \ldots,\left(x_{M}, t_{M}\right)$ where $\left(x_{j}, t_{j}\right) \in T, M \leqq N \tau^{-5 / 3}$ where $N$ is a constant that depends only on $\left\|w^{0}\right\|_{2}$, and the inequality $|(\operatorname{curl}(u))(x, t)| \leqq C \tau^{-2}$ holds whenever

$$
(x, t) \in T \sim \bigcup_{j=1}^{M}\left\{(y, s):\left|y-x_{j}\right| \leqq \tau, t_{j}-\tau^{2} \leqq s \leqq t_{j}\right\} .
$$

We use the notation introduced in Sect. 1 of [1]. The constant $C$ in the theorem does not depend on any of the parameters. Saying that $u$ is a weak solution means that $u$ satisfies the properties listed in Theorem 1.2 of [1] when the domain of $u$ is extended to all of $R^{3} \times(0, \infty)$, using the definition $u(x, t)=0$ for $x \notin U$. The condition $\operatorname{div}\left(w^{0}\right)=0$ means that $\int_{R^{3}} w^{0}(x) \cdot \nabla f(x) d x=0$ for any
$C^{\infty}$ function $f: R^{3} \rightarrow R$ with compact support.

In later sections we will use the definition

$$
(f * g)(x, t)=\int_{R^{3}} f(y, t) g(x-y) d y
$$

when $A \subset R, f$ is a function defined on $R^{3} \times A$, and $g$ is a function defined on $R^{3}$.

\section{Preliminaries}

The following definition is consistent with Definition 2.1 of [1].

Definition 2.1. If $A \subset R^{3} \times R$ and $f$ is a function defined on $A$, then we abbreviate $I(f, A)=\int_{A} f$ and $M(f, A)=$ the supremum of $\{|f(x, t)|:(x, t) \in A\}$. If $x \in R^{3}, t \in R, r>0, s>0, h>0$, and $k \in\{1,2,3, \ldots\}$, then

$$
\begin{aligned}
B(x, r) & =\left\{y \in R^{3}:|y-x| \leqq r\right\}, \\
K(x, t, r, s) & =\left\{(y, w) \in R^{3} \times R:|y-x| \leqq r \text { and } t-s \leqq w \leqq t\right\},
\end{aligned}
$$




$$
\begin{aligned}
T(x, t, r, s, h) & =\left\{(y, w) \in R^{3} \times R: r-h \leqq|y-x| \leqq r+h \text { and } t-s \leqq w \leqq t\right\}, \\
D(t) & =\left\{(y, w) \in R^{3} \times R: w \leqq t\right\} \\
G(x, t, r, k) & =K\left(x, t, r\left(1-2^{-k}\right), r^{2}\left(1-2^{-2 k}\right)\right) .
\end{aligned}
$$

Lemma 2.2. There exists an absolute constant $C_{1}$ with the following property: Suppose $u: R^{3} \times R^{+} \rightarrow R^{3}$ is a continuous function such that $\left\{\int_{R^{3}}|u(x, t)|^{2} d x: t>0\right\}$ is a bounded set of real numbers and $D u$ is an $L^{2}$ function. Suppose also that $\{a, c\} \subset R^{3},\{b, d\} \subset R,\{m, p, n, q\}$ is a set of integers, $b>2^{-2 m},|a-c|<2^{-m}, b$ $-2^{-2 m}<d \leqq b, 2^{-(n+1)}<2^{-m}-|a-c| \leqq 2^{-n}, 2^{-2(p+1)}<d-\left(b-2^{-2 m}\right) \leqq 2^{-2 p}, q$ $=\max \{n, p\}$, and $K=K\left(c, d, 2^{-m-2}, 2^{-2(p+2)}\right)$. Then

$$
p \geqq m, \quad n \geqq m
$$

and

$$
\begin{aligned}
& \int_{K}|u(x, t)|^{2}\left(|x-c|+(d-t)^{1 / 2}\right)^{-4} d x d t \\
& \leqq \sum_{k=m+1}^{q+1} C_{1} 2^{-k} M\left(|u|^{2}, D(d) \cap G\left(a, b, 2^{-m}, k-m+1\right)\right) \\
&+\sum_{k=m+1}^{q+1} C_{1} 2^{2 k} I\left(|D u|^{2}, T\left(a, b, 2^{-m}, 2^{-2 m}, 2^{-k+2}\right)\right) .
\end{aligned}
$$

Proof. The hypotheses of Sect. 2 of [1] are all satisfied. Hence Lemmas 2.2, 2.3, 2.4 of [1] are valid in this case. The second of these lemmas yields (2.1). Now we will use a portion of the proof of Lemma 2.5 of [1]. Using the definition of $E(k)$ given in $(2.30),(2.31)$ of [1] and (2.1), we obtain

$$
K \sim\{(c, d)\}=\bigcup_{k=m+2}^{\infty} E(k)
$$

From (2.33) of [1] we get

$$
\left(|x-c|+(d-t)^{1 / 2}\right)^{-4} \leqq 2^{4(k+1)} \quad \text { if }(x, t) \in E(k) .
$$

Combining (2.9) of [1], Definition 2.1, and the proof of Lemma 2.5 of [1], we find the following: If $|a-c| \leqq 2^{-(m+1)}$ then

$$
\sum_{k=m+2}^{\infty} 2^{4 k} I\left(|u|^{2}, E(k)\right) \leqq C 2^{-p} M\left(|u|^{2}, D(d) \cap G\left(a, b, 2^{-m}, p+2-m\right)\right) ;
$$

if $2^{-(m+1)}<|a-c|$ and $n \leqq p$ then

$$
\begin{aligned}
& \sum_{k=m+1}^{\infty} 2^{4 k} I\left(|u|^{2}, E(k)\right) \\
& \quad \leqq C 2^{-q} M\left(|u|^{2}, D(d) \cap G\left(a, b, 2^{-m}, q+2-m\right)\right) \\
& \quad+\sum_{k=m+1}^{q+1} C 2^{2 k} I\left(|D u|^{2}, T\left(a, b, 2^{-m}, 2^{-2 m}, 2^{-k+2}\right)\right)
\end{aligned}
$$


if $2^{-(m+1)}<|a-c|$ and $n>p$ then

$$
\begin{aligned}
\sum_{k=m+1}^{\infty} & 2^{4 k} I\left(|u|^{2}, E(k)\right) \\
\leqq & \sum_{k=m+1}^{q+1} C 2^{-k} M\left(|u|^{2}, D(d) \cap G\left(a, b, 2^{-m}, k+1-m\right)\right) \\
& \quad+\sum_{k=m+1}^{q+1} C 2^{2 k} I\left(|D u|^{2}, T\left(a, b, 2^{-m}, 2^{-2 m}, 2^{-k+2}\right)\right) .
\end{aligned}
$$

The conclusion follows from all of the above, $q=\max \{n, p\}$, and (2.1).

Lemma 2.3. Suppose $u: R^{3} \times R^{+} \rightarrow R^{3}$ is a continuous function of the type considered in Lemma 2.2. Suppose also that $\{a, c\} \subset R^{3},\{b, d\} \subset R, \tau>0, b>\tau^{2}, \mid a$ $-c \mid<\tau, b-\tau^{2}<d<b, n$ and $p$ are the integers determined uniquely by

$$
2^{-(n+1)}<\tau^{-1}(\tau-|a-c|) \leqq 2^{-n}, \quad 2^{-2(p+1)}<\tau^{-2}\left(d-\left(b-\tau^{2}\right)\right) \leqq 2^{-2 p},
$$

and $q=\max \{n, p\}, K=K\left(c, d, \tau / 4, \tau^{2} 2^{-2(p+2)}\right)$. Then

$$
p \geqq 0, \quad n \geqq 0
$$

and

$$
\begin{aligned}
& \int_{K}|u(x, t)|^{2}\left(|x-c|+(d-t)^{1 / 2}\right)^{-4} d x d t \\
& \leqq \sum_{k=1}^{q+1} C_{1} 2^{-k} \tau M\left(|u|^{2}, D(d) \cap G(a, b, \tau, k+1)\right) \\
& \quad+\sum_{k=1}^{q+1} C_{1} 2^{2 k} \tau^{-2} I\left(|D u|^{2}, T\left(a, b, \tau, \tau^{2}, \tau 2^{-k+2}\right)\right) .
\end{aligned}
$$

Proof. Set $a^{\prime}=\tau^{-1} a, c^{\prime}=\tau^{-1} c, b^{\prime}=\tau^{-2} b, d^{\prime}=\tau^{-2} d$, and $u^{\prime}(x, t)=u\left(\tau x, \tau^{2} t\right)$. The conclusion follows when $u, a, b, c, d$ in Lemma 2.2 are replaced by $u^{\prime}, a^{\prime}, b^{\prime}, c^{\prime}, d^{\prime}$ and the $m$ in that lemma is set equal to zero.

\section{An Integral Inequality for Approximate Solutions}

This section is a continuation of Sect. 5 of [1]. In particular, we have the choices of $\Phi, \Omega, \Psi$ made at the start of that section and we also have the function $u: R^{3} \times[0, \infty) \rightarrow R^{3}$ of Definition 5.14 of [1]. Lemma 5.6 and Definition 5.10 of [1] imply that $u$ is a function of the type considered in Lemma 2.2. Lemma 5.17 of [1] shows that $u$ is locally in $L^{3}$. The number $\varepsilon$ is introduced immediately after Definition 5.1 of [1]. We may assume that $\| \Delta(\Phi)$ $-\Delta(\Phi * \Omega * \Omega) \|_{2} \leqq \varepsilon$ holds instead of (5.1) of [1].

Lemma 3.1. There exists an absolute constant $C_{2}$ satisfying the following: If $\tau>0$ then there exists a continuous function $v: R^{3} \times R^{+} \rightarrow R^{3}$ such that $\left\{\int|v(x, t)|^{2} d x: t>0\right\}$ is a bounded set of real numbers, $D v$ is an $L^{2}$ function,

$$
\begin{gathered}
|v(x, t)| \leqq C_{2} \tau^{-3 / 2}\left(\int_{B(a, 5 \tau / 4)}|u(y, t)|^{2} d y\right)^{1 / 2} \quad \text { if } x \in B(a, \tau), \\
I\left(|D v|^{2}, T\left(a, b, \tau, \tau^{2}, r\right)\right) \leqq C_{2} \tau^{-4 / 3} r\left(I\left(|u|^{3}, K\left(a, b, 4 \tau, \tau^{2}\right)\right)\right)^{2 / 3}
\end{gathered}
$$


if $0<r \leqq 2 \tau$ and the following is true: If $a \in R^{3}, b \in R, \varepsilon<\tau / 64, B(a, 5 \tau / 4) \subset U$, $b>\tau^{2}, c \in R^{3}, d \in R,|a-c|<\tau, b-\tau^{2}<d<b, p$ and $n$ are integers defined by (2.2), $q=\max \{p, n\}$, and $A=K\left(a, b, 5 \tau / 4, \tau^{2}\right)$ then

$$
\begin{aligned}
\mid(u-v) & (c, d) \mid \\
\leqq & C_{2}\left(\int_{d-\tau^{2} 2^{-2(p+2)}}^{d} \int_{B(c, \tau / 4)}|(u-v)(x, t)|^{2}\left(|x-c|+(d-t)^{1 / 2}\right)^{-4} d x d t\right) \\
& +C_{2} \tau^{-2 / 3} M(|u-v|, D(d) \cap G(a, b, \tau, q+2))\left(\int_{A}|u(x, t)|^{3} d x d t\right)^{1 / 3} \\
& +C_{2} \tau^{-7 / 3} 2^{q}\left(\int_{A}|u(x, t)|^{3} d x d t\right)^{2 / 3} \\
& +C_{2} \tau^{-5 / 3} 2^{5 p / 3}\left(\int_{d-\tau^{2} 2^{-2(p+2)}}^{d} \int_{B(a, 5 \tau / 4)}|u(x, t)|^{3} d x d t\right)^{1 / 3} \\
& +C_{2} \tau^{2}\left\|w^{0}\right\|_{2} \varepsilon .
\end{aligned}
$$

Proof. Let $\lambda_{1}: R^{3} \rightarrow[0,1]$ be a $C^{\infty}$ function such that $\lambda_{1}(x)=0$ for $|x| \geqq \tau / 16$, $\lambda_{1}(x)=1$ for $|x| \leqq \tau / 32$, and $\left\|D^{i} \lambda_{1}\right\|_{\infty} \leqq C \tau^{-i}$ for $i=1,2,3$. Define $J: R^{3} \sim\{0\} \rightarrow R$ and $Q_{t}: R^{3} \rightarrow R$ for $t>0$ by

$$
J(x)=-(4 \pi|x|)^{-1}, \quad Q_{t}(x)=(2 \sqrt{\pi})^{-3} t^{-3 / 2} \exp \left(-|x|^{2} /(4 t)\right) .
$$

This is consistent with (5.6), (5.7) of [1]. The function $L: R^{3} \rightarrow R$ is given by

$$
L(x)=-\left[\Delta \lambda_{1}(-x) J(x)-2 D_{j} \lambda_{1}(-x) D_{j} J(x)\right] \text { for } x \neq 0,
$$

and $L(x)=0$ for $|x|<\tau / 32$. We define

$$
v=u * L
$$

and observe

$$
\begin{aligned}
|v(x, t)| \leqq C \tau^{-3 / 2}\left(\int_{B(a, 5 \tau / 4)}|u(y, t)|^{2} d y\right)^{1 / 2}, & \text { if } x \in B(a, 9 \tau / 8), \\
|D v(x, t)| \leqq C \tau^{-5 / 2}\left(\int_{B(a, 5 \tau / 4)}|u(y, t)|^{2} d y\right)^{1 / 2}, & \text { if } x \in B(a, 9 \tau / 8) .
\end{aligned}
$$

We abbreviate

$$
\begin{gathered}
K_{1}=K\left(c, d, \tau / 8, \tau^{2} 2^{-2(p+2)}\right), \quad K_{2}=K\left(c, d, \tau 2^{-(q+2)}, \tau^{2} 2^{-2(q+2)}\right), \\
h=\tau 2^{-(p+2)}, \quad H=\tau 2^{-(q+2)}, \quad B=B(a, 5 \tau / 4) .
\end{gathered}
$$

From $|c-a|<\tau, b>\tau^{2},(2.2)$, and $d<b$ we conclude

$$
B(c, \tau / 8) \subset B(a, 9 \tau / 8), \quad 0<b-\tau^{2}<d-h^{2}<d<b .
$$

If $x \in B\left(c, \tau 2^{-(q+2)}\right)$ then (2.2) and $q \geqq n$ imply

$$
\begin{aligned}
|x-a| & \leqq|x-c|+|c-a| \leqq \tau 2^{-(q+2)}+\tau-\tau 2^{-(n+1)} \\
& \leqq \tau 2^{-(q+2)}+\tau-\tau 2^{-(q+1)}=\tau\left(1-2^{-(q+2)}\right) .
\end{aligned}
$$


If $d-\tau^{2} 2^{-2(q+2)} \leqq t \leqq d$ then $q \geqq p,(2.2)$, and $d \leqq b$ imply

$$
\begin{aligned}
b-\tau^{2}\left(1-2^{-2(q+2)}\right) & \leqq b-\tau^{2}\left(1-2^{-2(p+2)}\right) \leqq d-\tau^{2} 2^{-2(p+2)} \\
& \leqq d-\tau^{2} 2^{-2(q+2)} \leqq t \leqq d \leqq b .
\end{aligned}
$$

All this implies

$$
K\left(c, d, \tau 2^{-(q+2)}, \tau^{2} 2^{-2(q+2)}\right) \subset D(d) \cap G(a, b, \tau, q+2) .
$$

In particular, we obtain

$$
B\left(c, \tau 2^{-(q+2)}\right) \subset B(a, \tau) \subset B(a, 9 \tau / 8) .
$$

We select a $C^{\infty}$ function $\lambda_{2}: R \rightarrow[0,1]$ such that $\lambda_{2}(t)=0$ for $t \leqq d-h^{2}, \lambda_{2}(t)=1$ for $t \geqq d-h^{2} / 2$, and $\left\|(d / d t) \lambda_{2}\right\|_{\infty} \leqq C \tau^{-2} 2^{2 p}$. Then we set $\lambda(x, t)=\lambda_{1}(x) \lambda_{2}(t)$.

Now we fix $i \in\{1,2,3\}$, recall (3.1), and define

$$
f: R^{3} \times(-\infty, d) \rightarrow R, \quad F: R^{3} \times(-\infty, d) \rightarrow R^{3}, \quad G: R^{3} \times(-\infty, d) \rightarrow R^{3}
$$

by

$$
\begin{aligned}
f(x, s) & =\lambda(x-c, s)\left(Q_{d-s} * J\right)(x-c), \\
F_{i}(x, s) & =f(x, s), \quad F_{j}(x, s)=0 \quad \text { if } j \in\{1,2,3\} \text { and } j \neq i, \\
G & =\operatorname{curl}(\operatorname{curl}(F)) .
\end{aligned}
$$

Using Definitions $5.1,5.2$ of $[1]$ and $\varepsilon<\tau / 64$ we obtain

$$
(G * \Psi * \Psi)(x, t)=0 \quad \text { if } x \notin B(c, \tau / 8) .
$$

The function $G$ satisfies

$$
\begin{aligned}
|G(x, t)| & \leqq C\left(|x-c|+(d-t)^{1 / 2}\right)^{-3}, \\
|D G(x, t)| & \leqq C\left(|x-c|+(d-t)^{1 / 2}\right)^{-4} .
\end{aligned}
$$

Using the proof of Lemma 5.7 of [1] we conclude

$$
\begin{array}{r}
|(G * \Psi * \Psi)(x, t)| \leqq C\left(|x-c|+(d-t)^{1 / 2}\right)^{-3} \\
|(D G * \Psi * \Psi)(x, t)| \leqq C\left(|x-c|+(d-t)^{1 / 2}\right)^{-4} .
\end{array}
$$

We also have

If $t<d$, then (3.12) yields

$$
\begin{aligned}
& \int_{d-h^{2}}^{d}\left(\int_{B(a, 5 \tau / 4)}|u(x, t)|^{2} d x\right)^{3 / 2} d t \\
& \leqq C \tau^{3 / 2}\left(\int_{d-h^{2}}^{d} \int_{B(a, 5 \tau / 4)}|u(x, t)|^{3} d x d t\right) .
\end{aligned}
$$

$$
\begin{aligned}
& \int_{B(a, 9 \tau / 8)}|(G * \Psi * \Psi)(x, t)| d x \\
& \leqq C \tau^{3 / 5}\left(\int_{B(a, 9 \tau / 8)}|(G * \Psi * \Psi)(x, t)|^{5 / 4} d x\right)^{4 / 5} \\
& \leqq C \tau^{3 / 5}\left(\int_{R^{3}}\left(|x-c|+(d-t)^{1 / 2}\right)^{-15 / 4} d x\right)^{4 / 5} \leqq C \tau^{3 / 5}(d-t)^{-3 / 10}
\end{aligned}
$$


Using (3.11), (3.7), (3.4), (3.6), (3.14), (3.13), and (3.7) we obtain

$$
\begin{aligned}
\int_{d-h^{2}}^{d} & \int_{R^{3}}|v(x, t)||D v(x, t)||(G * \Psi * \Psi)(x, t)| d x d t \\
& =\int_{d-h^{2}}^{d} \int_{B(a, 9 \tau / 8)}|v(x, t)||D v(x, t)||(G * \Psi * \Psi)(x, t)| d x d t \\
& \leqq C\left(\int_{-h^{2}}^{d} \tau^{-4}\left(\int_{B}|u(x, t)|^{2} d x\right)\left(\int_{B(a, 9 \tau / 8)}|(G * \Psi * \Psi)(x, t)| d x\right) d t\right) \\
\leqq & \\
& \leqq C \tau^{-17 / 5}\left(\int _ { d - h ^ { 2 } } ^ { d } \left(\int_{B}^{-17 / 5}\left(\int_{d-h^{2}}^{d}\left(\int_{B}|u(x, t)|^{2} d x\right)(d-t)^{-3 / 10} d t\right)\right.\right. \\
\leqq & \\
& \left.\leqq C \tau^{-12 / 5} h^{1 / 15}\left(\int_{d-h^{2}}^{d} \int_{B(a, 5 \tau / 4)}^{2} d x\right)^{3 / 2} d t\right)^{2 / 3}\left(\tau_{d-h^{2}}^{d}(d-t)^{-9 / 10} d t\right)^{1 / 3} \\
&
\end{aligned}
$$

From (3.5), (3.6), (3.12), (3.9), (3.4), $q \geqq p,(3.13)$, and (3.7) we conclude

$$
\begin{aligned}
\int_{K_{2}}|v(x, t)||(D G * \Psi * \Psi)(x, t)| d x d t \\
\quad \leqq \int_{d-H^{2}}^{d} \int_{B(c, H)} C|v(x, t)|\left(|x-c|+(d-t)^{1 / 2}\right)^{-4} d x d t \\
\quad \leqq \int_{d-H^{2}}^{d} C \tau^{-3 / 2}\left(\int_{B}|u(x, t)|^{2} d x\right)^{1 / 2}\left(\int_{B(c, H)}\left(|x-c|+(d-t)^{1 / 2}\right)^{-4} d x\right) d t \\
\leqq \int_{-H^{2}}^{d} C \tau^{-3 / 2}\left(\int_{B}|u(x, t)|^{2} d x\right)^{1 / 2}(d-t)^{-1 / 2} d t \\
\leqq C \tau^{-3 / 2}\left(\int_{d-H^{2}}^{d}\left(\int_{B}|u(x, t)|^{2} d x\right)^{3 / 2} d t\right)^{1 / 3}\left(\int_{d-H^{2}}^{d}(d-t)^{-3 / 4} d t\right)^{2 / 3} \\
\leqq C \tau^{-3 / 2} \tau^{1 / 2}\left(\int_{d-h^{2}}^{d} \int_{B}|u(x, t)|^{3} d x d t\right)^{1 / 3} H^{1 / 3} \\
\leqq C \tau^{-2 / 3} 2^{-q / 3}\left(\int_{b-\tau^{2}}^{b} \int_{B(a, 5 \tau / 4)}|u(x, t)|^{3} d x d t\right)^{1 / 3} .
\end{aligned}
$$

Using (3.11), (3.7), (3.4), (3.12), (3.6), (3.5), $q \geqq p$, and (3.7) we find

$$
\begin{aligned}
& \int_{K_{1} \sim K_{2}}|v(x, t)|^{2}|(D G * \Psi * \Psi)(x, t)| d x d t \\
& \quad \leqq \int_{K_{1} \sim K_{2}} C \tau^{-3}\left(\int_{B}|u(y, t)|^{2} d y\right)\left(|x-c|+(d-t)^{1 / 2}\right)^{-4} d x d t \\
& \quad \leqq \int_{d-h^{2}}^{d-H^{2}} C \tau^{-3}\left(\int_{B}|u(y, t)|^{2} d y\right)\left(\int_{R^{3}}\left(|x-c|+(d-t)^{1 / 2}\right)^{-4} d x\right) d t
\end{aligned}
$$




$$
\begin{aligned}
& +\int_{d-H^{2}}^{d} C \tau^{-3}\left(\int_{B}|u(y, t)|^{2} d y\right)\left(\int_{R^{3} \sim B(c, H)}\left(|x-c|+(d-t)^{1 / 2}\right)^{-4} d x\right) d t \\
\leqq & \int_{d-h^{2}}^{d-H^{2}} C \tau^{-3}\left(\int_{B}|u(y, t)|^{2} d y\right)(d-t)^{-1 / 2} d t \\
& +\int_{d-H^{2}}^{d} C \tau^{-3}\left(\int_{B}|u(y, t)|^{2} d y\right) H^{-1} d t \\
\leqq & \int_{d-h^{2}}^{d} C \tau^{-3}\left(\int_{B}|u(y, t)|^{2} d y \mid H^{-1} d t\right. \\
\leqq & C \tau^{-4} 2^{q}\left(\int_{b-\tau^{2}}^{b} \int_{B(a, 5 \tau / 4)}|u(y, t)|^{2} d y d t\right) \\
\leqq & C \tau^{-7 / 3} 2^{q}\left(\int_{b-\tau^{2}}^{b} \int_{B(a, 5 \tau / 4)}|u(x, t)|^{3} d x d t\right)^{2 / 3} .
\end{aligned}
$$

From (3.5), (3.6), (3.11), (3.8), (3.16), (3.12), and (3.17) we conclude

$$
\begin{aligned}
& \int_{d-h^{2}}^{d} \int_{R^{3}}|(u-v)(x, t)||v(x, t)||(D G * \Psi * \Psi)(x, t)| d x d t \\
& \leqq \\
& \quad \int_{K_{2}}|(u-v)(x, t)||v(x, t)||(D G * \Psi * \Psi)(x, t)| d x d t \\
& \quad+\left(\int_{K_{1} \sim K_{2}}|(u-v)(x, t)|^{2}|(D G * \Psi * \Psi)(x, t)| d x d t\right) \\
& \quad+\left(\int_{K_{1} \sim K_{2}}|v(x, t)|^{2}|(D G * \Psi * \Psi)(x, t)| d x d t\right) \\
& \leqq \\
& \quad M(|u-v|, D(d) \cap G(a, b, \tau, q+2)) C \tau^{-2 / 3} 2^{-q / 3}\left(\int_{b-\tau^{2}}^{b} \int_{B}|u(x, t)|^{3} d x d t\right)^{1 / 3} \\
& \quad+C\left(\int_{d-h^{2}}^{d} \int_{B(c, \tau / 4)}|(u-v)(x, t)|^{2}\left(|x-c|+(d-t)^{1 / 2}\right)^{-4} d x d t\right) \\
& \quad+C \tau^{-7 / 3} 2^{q}\left(\int_{b-\tau^{2}}^{b} \int_{B(a, 5 \tau / 4)}|u(x, t)|^{3} d x d t\right)^{2 / 3} .
\end{aligned}
$$

Finally, (3.11) and (3.12) yield

$$
\begin{aligned}
& \int_{d-h^{2}}^{d} \int_{R^{3}}|(u-v)(x, t)|^{2}|(D G * \Psi * \Psi)(x, t)| d x d t \\
& \leqq C\left(\int_{d-h^{2}}^{d} \int_{B(c, \tau / 4)}|(u-v)(x, t)|^{2}\left(|x-c|+(d-t)^{1 / 2}\right)^{-4} d x d t\right) .
\end{aligned}
$$

From Definitions 5.1, 5.2, 5.10, 5.14 and Lemma 5.6 of [1] we conclude

$$
u(x, t)=\left(w^{\prime}(t)\right)(x)=(w(t) * \Psi)(x), \operatorname{div}(w(t))=0, \quad \Psi(x)=\Psi(-x) .
$$

If $d-h^{2} / 2<s<d$ then (3.10), (3.20), the definition of $\lambda$ and Definition 5.5 of [1] yield 


$$
\begin{aligned}
\int_{R^{3}} & {\left[u_{i}(x, s)\right][\Delta f(x, s)] d x=\int_{R^{3}}\left[u_{k}(x, s)\right]\left[\Delta F_{k}(x, s)\right] d x } \\
= & -\int_{R^{3}}\left[u_{k}(x, s)\right]\left[G_{k}(x, s)\right] d x+\int_{R^{3}}\left[u_{k}(x, s)\right]\left[D_{k}(\operatorname{div}(F))(x, s)\right] d x \\
= & -\int_{R^{3}}\left[u_{k}(x, s)\right]\left[G_{k}(x, s)\right] d x=-\int_{R^{3}}\left[\left(w_{k}(s)\right)(x)\right]\left[\left(G_{k} * \Psi\right)(x, s)\right] d x \\
= & -\int_{d-h^{2}}^{s} \int_{R^{3}}\left[\left(\left(D_{t} w_{k}\right)(t)\right)(x)\right]\left[\left(G_{k} * \Psi\right)(x, t)\right] d x d t \\
& -\int_{d-h^{2}}^{s} \int_{R^{3}}\left[\left(w_{k}(t)\right)(x)\right]\left[\left(D_{t} G_{k} * \Psi\right)(x, t)\right] d x d t .
\end{aligned}
$$

Using Lemma 5.6 and Definition 5.2 of [1], $\operatorname{support}(G * \Psi) \subset U, \operatorname{div}(G * \Psi)=0$, and (3.20) we obtain

$$
\begin{aligned}
-\int_{R^{3}} & {\left[\left(D_{t} w_{k}(t)\right)(x)\right]\left[\left(G_{k} * \Psi\right)(x, t)\right] d x } \\
= & -\int_{R^{3}}\left[(P(S(w(t))))_{k}(x)\right]\left[\left(G_{k} * \Psi\right)(x, t)\right] d x \\
= & -\int_{R^{3}}\left[(S(w(t)))_{k}(x)\right]\left[\left(G_{k} * \Psi\right)(x, t)\right] d x \\
= & \int_{R^{3}}\left[\left(\left(\left(w_{j}^{\prime}(t)\right)\left(D_{j}\left(w_{k}^{\prime}(t)\right)\right)\right) * \Psi\right)(x)\right]\left[\left(G_{k} * \Psi\right)(x, t)\right] d x \\
& \quad-\int_{R^{3}}\left[\Delta\left(w_{k}(t) * \Omega * \Omega\right)(x)\right]\left[\left(G_{k} * \Psi\right)(x, t)\right] d x .
\end{aligned}
$$

Properties (3.20) and (3.10) give us

$$
\begin{aligned}
& -\int_{R^{3}}\left[\left(w_{k}(t)\right)(x)\right]\left[\left(D_{t} G_{k} * \Psi\right)(x, t)\right] d x \\
& -\int_{R^{3}}\left[\Delta\left(w_{k}(t) * \Omega * \Omega\right)(x)\right]\left[\left(G_{k} * \Psi\right)(x, t)\right] d x \\
& =-\int_{R^{3}}\left[\left(w_{k}(t)\right)(x)\right]\left[\left(D_{t} G_{k} * \Psi\right)(x, t)\right] d x \\
& \quad+\int_{R^{3}}\left[\left(w_{k}(t)\right)(x)\right]\left[\left(D_{t} D_{k}(\operatorname{div}(F)) * \Psi\right)(x, t)\right] d x \\
& \quad-\int_{R^{3}}\left[\Delta\left(w_{k}(t) * \Omega * \Omega\right)(x)\right]\left[\left(G_{k} * \Psi\right)(x, t)\right] d x \\
& \quad+\int_{R^{3}}\left[\Delta\left(w_{k}(t) * \Omega * \Omega\right)(x)\right]\left[\left(D_{k}(\operatorname{div}(F)) * \Psi\right)(x, t)\right] d x \\
& =\int_{R^{3}}\left[\left(w_{k}(t)\right)(x)\right]\left[\left(D_{t}\left(\Delta\left(F_{k}\right)\right) * \Psi\right)(x, t)\right] d x \\
& \quad+\int_{R^{3}}\left[\Delta\left(w_{k}(t) * \Omega * \Omega\right)(x)\right]\left[\left(\Delta\left(F_{k}\right) * \Psi\right)(x, t)\right] d x \\
& =\int_{R^{3}}\left[\left(w_{i}^{\prime}(t)\right)(x)\right]\left[\left(\left(D_{t}+\Delta\right)(\Delta f)\right)(x, t)\right] d x \\
& \quad+\int_{R^{3}}\left[\left(w_{i}(t) *(\Delta(\Psi * \Omega * \Omega)-\Delta(\Psi))\right)(x)\right][(\Delta f)(x, t)] d x .
\end{aligned}
$$


Observe that (3.10) and $\Delta\left(Q_{d-s} * J\right)=Q_{d-s}$ imply

$$
\left(\left(D_{t}+\Delta\right)(\Delta f)\right)(x, t)=0 \text { if }|x-c| \leqq \tau / 32 \text { and } d-h^{2} / 2 \leqq t<d .
$$

Using this and the fact $h<\tau$ (recall (3.6), (2.3)) we find

$$
\left\|\left(D_{t}+\Delta\right)(\Delta f)\right\|_{3 / 2} \leqq C h^{-5 / 3}=C \tau^{-5 / 3} 2^{5 p / 3} .
$$

The identity $\Delta\left(Q_{d-s} * J\right)=Q_{d-s}$ also yields

$$
\int_{R^{3}}|\Delta f(x, t)| d x \leqq C .
$$

From the assumptions made at the start of this section we conclude

$$
\begin{aligned}
\|\Delta(\Psi * \Omega * \Omega)-\Delta \Psi\|_{2} & =\|(\Delta(\Phi * \Omega * \Omega)-\Delta \Phi) * \Omega\|_{2} \\
& \leqq\|\Delta(\Phi * \Omega * \Omega)-\Delta \Phi\|_{2}\|\Omega\|_{1} \leqq \varepsilon
\end{aligned}
$$

Using (3.2), (3.3), the continuity of $u, \Delta\left(Q_{t} * J\right)=Q_{t},(3.21)-(3.23)$, (3.20), (3.7), (3.24), (3.26), (3.25), Lemma 5.6 of [1], and $h<\tau$ we find

$$
\begin{aligned}
&\left|u_{i}(c, d)-v_{i}(c, d)\right|\left|u_{i}(c, d)+\int_{R^{3}}\left[u_{i}(x, d)\right]\left[\Delta \lambda_{1}(x-c) J(x-c)+2 D_{j} \lambda_{1}(x-c) D_{j} J(x-c)\right] d x\right| \\
&= \lim _{s \rightarrow d^{-}}\left|\int_{R^{3}}\left[u_{i}(x, s)\right][\Delta f(x, s)] d x\right| \\
& \leqq\left|\int_{d-h^{2}}^{d} \int_{R^{3}}\left[\left(\left(\left(w_{j}^{\prime}(t)\right)\left(D_{j}\left(w_{k}^{\prime}(t)\right)\right)\right) * \Psi\right)(x)\right]\left[\left(G_{k} * \Psi\right)(x, t)\right] d x d t\right| \\
&+\int_{d-h^{2}}^{d}\left|\left(w_{R^{3}}^{\prime}(t)\right)(x)\right|\left|\left(\left(D_{t}+\Delta\right)(\Delta f)\right)(x, t)\right| d x d t \\
&+\int_{d-h^{2}}^{d} \int_{R^{3}}\left|\left(w_{i}(t) *(\Delta(\Psi * \Omega * \Omega)-\Delta \Psi)\right)(x)\right||(\Delta f)(x, t)| d x d t \\
& \leqq \int_{d-h^{2}}^{d} \int_{R^{3}}\left[\left(w_{j}^{\prime}(t)\right)(x)\right]\left[D_{j}\left(w_{k}^{\prime}(t)\right)(x)\right]\left[\left(G_{k} * \Psi * \Psi\right)(x, t)\right] d x d t \mid \\
&\left.+C \int_{d-h^{2}}^{d} \int_{B(a, 5 \tau / 4)}|u(x, t)|^{3} d x d t\right)^{1 / 3} \tau^{-5 / 3} 2^{5 p / 3} \\
&+\int_{d-h^{2}}^{d}\|w(t)\|_{2}\|\Delta(\Psi * \Omega * \Omega)-\Delta \Psi\|_{2}\left(\int|\Delta f(x, t)| d x\right) d t \\
& \leqq \int_{d-h^{2}}^{d} \int_{R^{3}}\left[u_{j}(x, t)\right]\left[D_{j} u_{k}(x, t)\right]\left[\left(G_{k} * \Psi * \Psi\right)(x, t)\right] d x d t \mid \\
&\left.+C \int_{d-h^{2}}^{d} \int_{B(a, 5 \tau / 4)}|u(x, t)|^{3} d x d t\right)^{1 / 3} \tau^{-5 / 3} 2^{5 p / 3}+C \tau^{2}\left\|w^{0}\right\|_{2} \varepsilon . \\
&=
\end{aligned}
$$

From (3.20) and (3.3) we conclude $\operatorname{div}(u)=0, \operatorname{div}(v)=0$. Hence (3.19), (3.18), and (3.15) yield 


$$
\begin{aligned}
& \left|\int_{d-h^{2}}^{d} \int_{R^{3}}\left[u_{j}(x, t)\right]\left[D_{j} u_{k}(x, t)\right]\left[\left(G_{k} * \Psi * \Psi\right)(x, t)\right] d x d t\right| \\
& \leqq \int_{d-h^{2}}^{d}\left|\int\left[\left(u_{R^{3}}-v_{j}\right)(x, t)\right]\left[D_{j}\left(u_{k}-v_{k}\right)(x, t)\right]\left[\left(G_{k} * \Psi * \Psi\right)(x, t)\right] d x\right| d t \\
& +\int_{d-h^{2}}^{d}\left|\int_{R^{3}}\left[v_{j}(x, t)\right]\left[D_{j}\left(u_{k}-v_{k}\right)(x, t)\right]\left[\left(G_{k} * \Psi * \Psi\right)(x, t)\right] d x\right| d t \\
& +\int_{d-h^{2}}^{d}\left|\int_{R^{3}}\left[\left(u_{j}-v_{j}\right)(x, t)\right]\left[D_{j} v_{k}(x, t)\right]\left[\left(G_{k} * \Psi * \Psi\right)(x, t)\right] d x\right| d t \\
& +\int_{d-h^{2}}^{d}\left|\int_{R^{3}}\left[v_{j}(x, t)\right]\left[D_{j} v_{k}(x, t)\right]\left[\left(G_{k} * \Psi * \Psi\right)(x, t)\right] d x\right| d t \\
& \leqq \int_{d-h^{2}}^{d}\left|\int_{R^{3}}\left[\left(u_{j}-v_{j}\right)(x, t)\right]\left[\left(u_{k}-v_{k}\right)(x, t)\right]\left[\left(D_{j} G_{k} * \Psi * \Psi\right)(x, t)\right] d x\right| d t \\
& +\int_{d-h^{2}}^{d}\left|\int_{R^{3}}\left[v_{j}(x, t)\right]\left[\left(u_{k}-v_{k}\right)(x, t)\right]\left[\left(D_{j} G_{k} * \Psi * \Psi\right)(x, t)\right] d x\right| d t \\
& +\int_{d-h^{2}}^{d}\left|\int_{R^{3}}\left[\left(u_{j}-v_{j}\right)(x, t)\right]\left[v_{k}(x, t)\right]\left[\left(D_{j} G_{k} * \Psi * \Psi\right)(x, t)\right] d x\right| d t \\
& +\int_{d-h^{2}}^{d}\left|\int_{R^{3}}\left[v_{j}(x, t)\right]\left[D_{j} v_{k}(x, t)\right]\left[\left(G_{k} * \Psi * \Psi\right)(x, t)\right] d x\right| d t \\
& \leqq C\left(\int_{d-h^{2}}^{d} \int_{B(c, \tau / 4)}|(u-v)(x, t)|^{2}\left(|x-c|+(d-t)^{1 / 2}\right)^{-4} d x d t\right) \\
& +M(|u-v|, D(d) \cap G(a, b, \tau, q+2)) C \tau^{-2 / 3} 2^{-q / 3}\left(\int_{b-\tau^{2}}^{b} \int_{B}|u(x, t)|^{3} d x d t\right)^{1 / 3} \\
& +C \tau^{-7 / 3} 2^{q}\left(\int_{b \sim \tau^{2}}^{b} \int_{B(a, 5 \tau / 4)}|u(x, t)|^{3} d x d t\right)^{2 / 3} \\
& +C \tau^{-7 / 3} 2^{-p / 15}\left(\int_{b-\tau^{2}}^{b} \int_{B(a, 5 \tau / 4)}|u(x, t)|^{3} d x d t\right)^{2 / 3} .
\end{aligned}
$$

The last inequality of the lemma follows from the above, (3.27), and $q \geqq p \geqq 0$ (see (2.3)). The estimate on $v$ follows from (3.4). The argument that gave us (3.4) yields the following for $0<r \leqq 2 \tau$ :

$$
\begin{aligned}
I(\mid D & \left.\left.v\right|^{2}, T\left(a, b, \tau, \tau^{2}, r\right)\right) \\
& \leqq \int_{T\left(a, b, \tau, \tau^{2}, r\right)}\left(C \tau^{-5 / 2}\left(\int_{B(a, 4 \tau)}|u(y, t)|^{2} d y\right)^{1 / 2}\right)^{2} d x d t \\
& \leqq \int_{b-\tau^{2}}^{b} \operatorname{Cr} \tau^{-3}\left(\int_{B(a, 4 \tau)}|u(y, t)|^{2} d y\right) d t \\
& \leqq C r \tau^{-4 / 3}\left(\int_{-\tau^{2}}^{b} \int_{B(a, 4 \tau)}|u(y, t)|^{3} d y d t\right)^{2 / 3}
\end{aligned}
$$

This gives us the estimate on $D v$. 


\section{An Estimate for Approximate Solutions}

The assumptions of Sect. 3 are still in force.

Lemma 4.1. There exist absolute constants $C_{3}, C_{4}$ with the following property: Suppose $\tau>0, v$ is the function of Lemma 3.1, $a \in R^{3}, \quad b \in R, \varepsilon<\tau / 64$, $B(a, 5 \tau / 4) \subset U$, and $b>\tau^{2}$. Suppose also that

$$
\begin{gathered}
I\left(|D u|^{2}, T\left(a, b, \tau, \tau^{2}, r\right)\right) \leqq C_{3} r \quad \text { if } 0<r \leqq 2 \tau, \\
I\left(|u|^{3}, K\left(a, b-\tau^{2}+s, 4 \tau, s\right)\right) \leqq C_{3} s \quad \text { if } 0<s \leqq \tau^{2}, \\
\left\|w^{0}\right\|_{2} \leqq C_{3} \tau^{-3} \varepsilon^{-1} .
\end{gathered}
$$

Then

$$
|(u-v)(x, t)| \leqq 2 C_{4} \tau^{-1} \quad \text { if }(x, t) \in K\left(a, b, \tau / 2,3 \tau^{2} / 4\right) .
$$

Proof. We choose $C_{4}>0$ so that $16 C_{2} C_{1} C_{4}^{2} \leqq(1 / 4) C_{4}$. Then we choose $C_{3}>0$ so that

$32 C_{1} C_{2} C_{3}+32 C_{1} C_{2}^{2} C_{3}^{2 / 3}+4 C_{2} C_{3}^{1 / 3} C_{4}+C_{2} C_{3}^{2 / 3}+C_{2} C_{3}^{1 / 3}+C_{2} C_{3} \leqq(1 / 4) C_{4}$.

We will use the method in the proof of Lemma 3.1 of [1]. A slight modification of the construction in that lemma gives us a continuous function

$$
f: \text { interior }\left(K\left(a, b, \tau, \tau^{2}\right)\right) \rightarrow R^{+}
$$

such that (recall Definition 2.1)

$$
C_{4} 2^{i} \tau^{-1} \geqq f(x, t) \geqq C_{4} 2^{i-1} \tau^{-1} \quad \text { if }(x, t) \in G(a, b, \tau, i) \sim G(a, b, \tau, i-1)
$$

for $i=1,2,3, \ldots$. Here we define $G(a, b, \tau, 0)$ to be the empty set. In particular, we get

$$
f(x, t) \leqq C_{4} 2^{i} \tau^{-1} \quad \text { if }(x, t) \in G(a, b, \tau, i) .
$$

We intend to show

$$
|(u-v)(x, t)| \leqq f(x, t) \quad \text { for all }(x, t) \in \text { interior }\left(K\left(a, b, \tau, \tau^{2}\right)\right) .
$$

Assume that (4.7) is false. Then the nature of $f$ and the continuity of $f$ and $u$ $-v$ imply the existence of $(c, d) \in$ interior $\left(K\left(a, b, \tau, \tau^{2}\right)\right)$ such that

$$
\begin{gathered}
|(u-v)(c, d)|=f(c, d), \\
|(u-v)(x, t)| \leqq f(x, t) \quad \text { if }(x, t) \in D(d) \cap \text { interior }\left(K\left(a, b, \tau, \tau^{2}\right)\right) .
\end{gathered}
$$

Using this $(c, d)$ we define the integers $p, n, q$ by (2.2) and $q=\max \{p, n\}$. From (2.2) and (4.2) we obtain

$$
\begin{aligned}
& I\left(|u|^{3}, K\left(a, d, 5 \tau / 4, \tau^{2} 2^{-2(p+2)}\right)\right) \\
& \quad \leqq I\left(|u|^{3}, K\left(a, d, 5 \tau / 4, d-\left(b-\tau^{2}\right)\right) \leqq C_{3}\left(d-\left(b-\tau^{2}\right)\right) \leqq C_{3} \tau^{2} 2^{-2 p} .\right.
\end{aligned}
$$


We can use Lemma 3.1, (4.1), and (4.2) to conclude the following for $k \geqq 1$ :

$$
\begin{aligned}
& I\left(|D(u-v)|^{2}, T\left(a, b, \tau, \tau^{2}, \tau 2^{-k+2}\right)\right) \\
& \quad \leqq 2 I\left(|D u|^{2}, T\left(a, b, \tau, \tau^{2}, \tau 2^{-k+2}\right)\right)+2 I\left(|D v|^{2}, T\left(a, b, \tau, \tau^{2}, \tau 2^{-k+2}\right)\right) \\
& \quad \leqq 2 C_{3} \tau 2^{-k+2}+2 C_{2} \tau^{-4 / 3} \tau 2^{-k+2}\left(I\left(|u|^{3}, K\left(a, b, 4 \tau, \tau^{2}\right)\right)\right)^{2 / 3} \\
& \quad \leqq 2 C_{3} \tau 2^{-k+2}+2 C_{2} C_{3}^{2 / 3} \tau 2^{-k+2} .
\end{aligned}
$$

Since the definitions of $p, n, q$ imply $(c, d) \notin$ interior $(G(a, b, \tau, q))$, we find that (4.5), (4.8) yield

$$
|(u-v)(c, d)|=f(c, d) \geqq C_{4} 2^{q} \tau^{-1} .
$$

Combining (4.12), Lemma 3.1, Lemma 2.3 with $u-v$ in place of $u,(4.2),(4.10)$, (4.9), (4.6), (4.3), (4.11), (2.3), $q \geqq p$, and the definitions of $C_{3}, C_{4}$ we find

$$
\begin{aligned}
& C_{4} 2^{q} \tau^{-1} \leqq|(u-v)(c, d)| \\
& \leqq C_{2}\left(\int_{d-\tau^{2} 2^{-2(p+2)}}^{d} \int_{B(c, \tau / 4)}|(u-v)(x, t)|^{2}\left(|x-c|+(d-t)^{1 / 2}\right)^{-4} d x d t\right) \\
& +C_{2} \tau^{-2 / 3} M(|u-v|, D(d) \cap G(a, b, \tau, q+2))\left(C_{3} \tau^{2}\right)^{1 / 3} \\
& +C_{2} \tau^{-7 / 3} 2^{q}\left(C_{3} \tau^{2}\right)^{2 / 3}+C_{2} \tau^{-5 / 3} 2^{5 p / 3}\left(C_{3} \tau^{2} 2^{-2 p}\right)^{1 / 3}+C_{2} \tau^{2}\left\|w^{0}\right\|_{2} \varepsilon \\
& \leqq \sum_{k=1}^{q+1} C_{2} C_{1} 2^{-k} \tau M\left(|u-v|^{2}, D(d) \cap G(a, b, \tau, k+1)\right) \\
& +\sum_{k=1}^{q+1} C_{2} C_{1} 2^{2 k} \tau^{-2} I\left(|D(u-v)|^{2}, T\left(a, b, \tau, \tau^{2}, \tau 2^{-k+2}\right)\right) \\
& +C_{2} \tau^{-2 / 3}\left(C_{4} 2^{q+2} \tau^{-1}\right)\left(C_{3} \tau^{2}\right)^{1 / 3}+C_{2} C_{3}^{2 / 3} \tau^{-1} 2^{q}+C_{2} C_{3}^{1 / 3} \tau^{-1} 2^{p}+C_{2} C_{3} \tau^{-1} \\
& \leqq \sum_{k=1}^{q+1} C_{2} C_{1} 2^{-k} \tau\left(C_{4} 2^{k+1} \tau^{-1}\right)^{2}+\sum_{k=1}^{q+1} C_{2} C_{1} 2^{2 k} \tau^{-2}\left(2 C_{3} \tau 2^{-k+2}+2 C_{2} C_{3}^{2 / 3} \tau 2^{-k+2}\right) \\
& +4 C_{2} C_{4} C_{3}^{1 / 3} \tau^{-1} 2^{q}+C_{2} C_{3}^{2 / 3} \tau^{-1} 2^{q}+C_{2} C_{3}^{1 / 3} \tau^{-1} 2^{q}+C_{2} C_{3} \tau^{-1} 2^{q} \\
& \leqq 16 C_{2} C_{1} C_{4}^{2} \tau^{-1} 2^{q}+32 C_{2} C_{1} C_{3} \tau^{-1} 2^{q}+32 C_{2}^{2} C_{1} C_{3}^{2 / 3} \tau^{-1} 2^{q}+4 C_{2} C_{4} C_{3}^{1 / 3} \tau^{-1} 2^{q} \\
& +C_{2} C_{3}^{2 / 3} \tau^{-1} 2^{q}+C_{2} C_{3}^{1 / 3} \tau^{-1} 2^{q}+C_{2} C_{3} \tau^{-1} 2^{q} \\
& \leqq(1 / 4) C_{4} \tau^{-1} 2^{q}+(1 / 4) C_{4} \tau^{-1} 2^{q}=(1 / 2) C_{4} \tau^{-1} 2^{q} \text {. }
\end{aligned}
$$

This is a contradiction, hence we cannot assume that (4.7) is false. The conclusion follows from (4.7), (4.6), and the definition of $G(a, b, \tau, i)$.

Lemma 4.2. There exist absolute constants $C_{5}, C_{6}$ with the following property: Suppose $\sigma>0, a \in R^{3}, b \in R, \varepsilon<\sigma / 128, B(a, 5 \sigma / 4) \subset U$, and $b>\sigma^{2}$. Suppose also that

$$
\begin{gathered}
I\left(|D u|^{2}, K\left(a, b, 3 \sigma, \sigma^{2}\right)\right) \leqq C_{5} \sigma, \\
I\left(|u|^{3}, K\left(a, b, 4 \sigma, \sigma^{2}\right)\right) \leqq C_{5} \sigma^{2} \\
\left\|w^{0}\right\|_{2} \leqq C_{5} \sigma^{-3} \varepsilon^{-1}
\end{gathered}
$$


Then

$$
\int_{b-3 \sigma^{2} / 16}^{b}(\max \{|u(x, t)|: x \in B(a, \sigma / 4)\})^{3} d t \leqq C_{6} \sigma^{-1} .
$$

Proof. Using the method in Sect. 4 of [1] (which is an application of the Hardy-Littlewood weak-type inequality for $L^{1}$ ), we can find $C_{5} \leqq C_{3}$ such that (4.13), (4.14) imply that (4.1), (4.2) hold for some $\tau$ such that $\sigma / 2<\tau<\sigma$. Using Lemma 4.1 we obtain (4.4) and hence

$$
|(u-v)(x, t)| \leqq 4 C_{4} \sigma^{-1} \quad \text { if }(x, t) \in K\left(a, b, \sigma / 4,3 \sigma^{2} / 16\right),
$$

where $v$ is the function corresponding to $\tau$ in Lemma 3.1. From (4.17), Lemma 3.1, $\sigma / 2<\tau<\sigma$, Hölder's inequality and (4.14) we get

$$
\begin{aligned}
& \int_{b-3 \sigma^{2} / 16}^{b}(\max \{|u(x, t)|: x \in B(a, \sigma / 4)\})^{3} d t \\
& \leqq \int_{b-3 \sigma^{2} / 16}^{b}\left(4 C_{4} \sigma^{-1}+C_{2}(\sigma / 2)^{-3 / 2}\left(\int_{B(a, 5 \sigma / 4)}|u(x, t)|^{2} d x\right)^{1 / 2}\right)^{3} d t \\
& \leqq C \sigma^{-1}+C \sigma^{-3}\left(\int_{b-3 \sigma^{2} / 16}^{b} \int_{B(a, 5 \sigma / 4)}|u(x, t)|^{3} d x d t\right) \leqq C \sigma^{-1} .
\end{aligned}
$$

\section{An Integral Inequality for the Vorticity of Approximate Solutions}

We continue with the same assumptions made in Sect.3. Recalling Definition 5.14 of [1], we have

$$
z=\operatorname{curl}(u)
$$

Lemma 5.1. There exists an absolute constant $C_{7}$ with the following property: Suppose $a \in R^{3}, b \in R, \tau>0, B(a, 5 \tau / 4) \subset U, b>\tau^{2}, \varepsilon<\tau / 64$,

$$
M(t)=\max \{|u(x, t)|: x \in B(a, 5 \tau / 4)\},
$$

$|a-c|<\tau, b-\tau^{2}<d<b$, and $n, p, q$ are defined as in Lemma 2.3. Then

$$
\begin{aligned}
|z(c, d)| \leqq & C_{7} \tau^{1 / 3} M(|z|, D(d) \cap G(a, b, \tau, q+2))\left(\int_{b-\tau^{2}}^{b} M(t)^{3} d t\right)^{1 / 3} \\
& +\sum_{k=p+2}^{q+1} C_{7} \tau^{-3} 2^{3 k} I\left(|D u|^{2}, T\left(a, b, \tau, \tau^{2}, \tau 2^{-k+2}\right)\right) \\
& +C_{7} \tau^{-4 / 3} 2^{2 q}\left(\int_{b-\tau^{2}}^{b} M(t)^{3} d t\right)^{2 / 3} \\
& +C_{7} \tau^{-3} 2^{3 p}\left(\int_{d-\tau^{2} 2^{2(p+2)}}^{d} \int_{B(a, 5 \tau / 4)}|D u(x, t)|^{2} d x d t\right) \\
& +C_{7} \tau^{-5 / 2} 2^{5 p / 2}\left(\int_{d-\tau^{2} 2^{-2(p+2)}}^{d} \int_{B(a, 5 \tau / 4)}|D u(x, t)|^{2} d x d t\right)^{1 / 2} \\
& +C_{7}\left\|w^{0}\right\|_{2} \tau \varepsilon .
\end{aligned}
$$


Proof. Let $h=\tau 2^{-(p+2)}, H=\tau 2^{-(q+2)}$,

$$
\begin{gathered}
K_{1}=K\left(c, d, \tau 2^{-(p+2)}, \tau^{2} 2^{-2(p+2)}\right), \\
K_{2}=K\left(c, d, \tau 2^{-(q+2)}, \tau^{2} 2^{-2(q+2)}\right), \\
K_{3}=K\left(c, d, \tau / 4, \tau^{2} 2^{-2(p+2)}\right), \\
E(k)=K\left(c, d, \tau 2^{-k}, \tau^{2} 2^{-2 k}\right) \sim K\left(c, d, \tau 2^{-(k+1)}, \tau^{2} 2^{-2(k+1)}\right)
\end{gathered}
$$

if $k$ is an integer and $k \geqq p+2$. The argument that led to (3.8) still applies, so we have

$$
K_{2} \subset D(d) \cap G(a, b, \tau, q+2) .
$$

As in Lemma 2.3, we can still say

$$
p \geqq 0, \quad n \geqq 0 .
$$

Now we will prove (recall Definition 2.1)

$$
K\left(c, d, \tau 2^{-k}, \tau^{2} 2^{-2 k}\right) \subset T\left(a, b, \tau, \tau^{2}, \tau 2^{-k+2}\right) \quad \text { if } p+2 \leqq k \leqq n+1
$$

and $k$ is an integer. If $x \in B\left(c, \tau 2^{-k}\right)$ and $k \leqq n+1$ then

$$
\begin{aligned}
& |x-a| \leqq|c-a|+|x-c|<\tau+\tau 2^{-k}<\tau+\tau 2^{-k+2}, \\
& |x-a| \geqq|c-a|-|x-c| \geqq \tau-\tau 2^{-n}-\tau 2^{-k} \geqq \tau-\tau 2^{-k+1}-\tau 2^{-k}>\tau-\tau 2^{-k+2} .
\end{aligned}
$$

This implies

$$
B\left(c, \tau 2^{-k}\right) \subset\left\{x \in R^{3}: \tau-\tau 2^{-k+2} \leqq|x-a| \leqq \tau+\tau 2^{-k+2}\right\} \quad \text { if } k \leqq n+1 .
$$

The argument that gave (3.7) is still valid, so we have

$$
0<b-\tau^{2}<d-\tau^{2} 2^{-2(p+2)}<d<b .
$$

Combining (5.5), (5.6) we get (5.4). In addition, we get

$$
\left(|x-c|+(d-t)^{1 / 2}\right)^{-3} \leqq \tau^{-3} 2^{3(k+1)} \quad \text { if }(x, t) \in E(k) .
$$

Using (5.2), (5.6), and $q \geqq p$ we find

$$
\begin{aligned}
\int_{K_{2}}|u(x, t)||z(x, t)|\left(|x-c|+(d-t)^{1 / 2}\right)^{-4} d x d t \\
\leqq M\left(|z|, K_{2}\right)\left(\int_{d-H^{2}}^{d}\left(M(t) \int_{B(c, H)}\left(|x-c|+(d-t)^{1 / 2}\right)^{-4} d x\right) d t\right) \\
\leqq M\left(|z|, K_{2}\right) C\left(\int_{d-H^{2}}^{d} M(t)(d-t)^{-1 / 2} d t\right) \\
\leqq M\left(|z|, K_{2}\right) C\left(\int_{d-H^{2}}^{d} M(t)^{3} d t\right)^{1 / 3}\left(\int_{d-H^{2}}^{d}(d-t)^{-3 / 4} d t\right)^{2 / 3}
\end{aligned}
$$




$$
\begin{aligned}
& \leqq M\left(|z|, K_{2}\right) C\left(\int_{b-\tau^{2}}^{b} M(t)^{3} d t\right)^{1 / 3} H^{1 / 3} \\
& \leqq C \tau^{1 / 3} 2^{-q / 3} M(|z|, D(d) \cap G(a, b, \tau, q+2))\left(\int_{b-\tau^{2}}^{b} M(t)^{3} d t\right)^{1 / 3} .
\end{aligned}
$$

If $q>p$ then $q=n>p$, so (5.7) and (5.4) yield

$$
\begin{aligned}
& \int_{K_{1} \sim K_{2}}|z(x, t)|^{2}\left(|x-c|+(d-t)^{1 / 2}\right)^{-3} d x d t \\
& \quad \leqq \sum_{k=p+2}^{q+1} I\left(|z|^{2}, E(k)\right) \tau^{-3} 2^{3(k+1)} \\
& \quad \leqq \sum_{k=p+2}^{q+1} C \tau^{-3} 2^{3 k} I\left(|D u|^{2}, T\left(a, b, \tau, \tau^{2}, \tau 2^{-k+2}\right)\right) .
\end{aligned}
$$

From (5.3) and $|a-c|<\tau$ we conclude $B\left(c, \tau 2^{-(p+2)}\right) \subset B(a, 5 \tau / 4)$. Using this and (5.6) we conclude the following when $q>p$ :

$$
\begin{aligned}
& \int_{K_{1} \sim K_{2}}|u(x, t)|^{2}\left(|x-c|+(d-t)^{1 / 2}\right)^{-5} d x d t \\
& \leqq \int_{d-h^{2}}^{d-H^{2}}\left(M(t)^{2} \int_{R^{3}}\left(|x-c|+(d-t)^{1 / 2}\right)^{-5} d x\right) d t \\
& \quad+\int_{d-H^{2}}^{d}\left(M(t)^{2} \int_{R^{3} \sim B(c, H)}\left(|x-c|+(d-t)^{1 / 2}\right)^{-5} d x\right) d t \\
& \leqq\left.C \int_{d-h^{2}}^{d-H^{2}} M(t)^{2}(d-t)^{-1} d t\right)+C\left(\int_{d-H^{2}}^{d} M(t)^{2} H^{-2} d t\right) \\
& \leqq C\left(\int_{d-h^{2}}^{d} M(t)^{2} H^{-2} d t\right) \leqq C H^{-2}\left(\int_{b-\tau^{2}}^{b} M(t)^{2} d t\right) \\
& \leqq C \tau^{-4 / 3} 2^{2 q}\left(\int_{b-\tau^{2}}^{b} M(t)^{3} d t\right)^{2 / 3} .
\end{aligned}
$$

If $q=p$ then $K_{1} \sim K_{2}$ is the empty set. Hence we can use (5.8)-(5.10) and the inequality

$$
\begin{aligned}
& |u(x, t)||z(x, t)|\left(|x-c|+(d-t)^{1 / 2}\right)^{-4} \\
& \quad \leqq(1 / 2)|u(x, t)|^{2}\left(|x-c|+(d-t)^{1 / 2}\right)^{-5}+(1 / 2)|z(x, t)|^{2}\left(|x-c|+(d-t)^{1 / 2}\right)^{-3}
\end{aligned}
$$

to conclude

$$
\begin{aligned}
& \int_{K_{1}}|u(x, t)||z(x, t)|\left(|x-c|+(d-t)^{1 / 2}\right)^{-4} d x d t \\
& \leqq \\
& \quad C \tau^{1 / 3} 2^{-q / 3} M(|z|, D(d) \cap G(a, b, \tau, q+2))\left(\int_{b-\tau^{2}}^{b} M(t)^{3} d t\right)^{1 / 3} \\
& \quad+\sum_{k=p+2}^{q+1} C \tau^{-3} 2^{3 k} I\left(|D u|^{2}, T\left(a, b, \tau, \tau^{2}, \tau 2^{-k+2}\right)\right) \\
& \quad+C \tau^{-4 / 3} 2^{2 q}\left(\int_{b-\tau^{2}}^{b} M(t)^{3} d t\right)^{2 / 3} .
\end{aligned}
$$


If $q=p$ then the second term in the right hand side of (5.11) is, of course, zero. Using $|c-a|<\tau$ and (5.6) we find

$$
\begin{aligned}
& \int_{K_{3} \sim K_{1}}|u(x, t)||z(x, t)|\left(|x-c|+(d-t)^{1 / 2}\right)^{-4} d x d t \\
& \leqq \int_{K_{3} \sim K_{1}}|u(x, t)|^{2}\left(|x-c|+(d-t)^{1 / 2}\right)^{-5} d x d t \\
&+\int_{K_{3} \sim K_{1}}|z(x, t)|^{2}\left(|x-c|+(d-t)^{1 / 2}\right)^{-3} d x d t \\
& \leqq \int_{d-h^{2}}^{d}\left(M(t)^{2} \int_{R^{3} \sim B(c, h)}\left(|x-c|+(d-t)^{1 / 2}\right)^{-5} d x\right) d t \\
&+C\left(\int_{K_{3}}|D u(x, t)|^{2} h^{-3} d x d t\right) \\
& \leqq C\left(\int_{d-h^{2}}^{d} M(t)^{2} h^{-2} d t\right)+C h^{-3}\left(\int_{K_{3}}|D u(x, t)|^{2} d x d t\right) \\
& \leqq C \tau^{-4 / 3} 2^{4 p / 3}\left(\int_{d-h^{2}}^{d} M(t)^{3} d t\right)^{2 / 3}+C \tau^{-3} 2^{3 p}\left(\int_{K_{3}}|D u(x, t)|^{2} d x d t\right) \\
& \leqq C \tau^{-4 / 3} 2^{4 p / 3}\left(\int_{b-\tau^{2}}^{b} M(t)^{3} d t\right)^{2 / 3} \\
&+C \tau^{-3} 2^{3 p}\left(\int_{d-h^{2} B(a, 5 \tau / 4)}^{d}|D u(x, t)|^{2} d x d t\right) .
\end{aligned}
$$

Now let $\lambda_{1}: R^{3} \rightarrow[0,1]$ be a $C^{\infty}$ function such that $\lambda_{1}(x)=0$ for $|x-c|$ $\geqq \tau / 16, \lambda_{1}(x)=1$ for $|x-c| \leqq \tau / 32$, and $\left\|D^{i} \lambda_{1}\right\|_{\infty} \leqq C \tau^{-i}$ for $i=1,2$. Let $\lambda_{2}$ : $R \rightarrow[0,1]$ be a $C^{\infty}$ function such that $\lambda_{2}(t)=0$ for $t \leqq d-h^{2}, \lambda_{2}(t)=1$ for $t \geqq d$ $-h^{2} / 2$, and $\left\|(d / d t) \lambda_{2}\right\|_{\infty} \leqq C h^{-2}$. We set $\lambda(x, t)=\lambda_{1}(x) \lambda_{2}(t)$, fix $i \in\{1,2,3\}$, and define $f: R^{3} \times(-\infty, d) \rightarrow R, g: R^{3} \times(-\infty, d) \rightarrow R^{3}$ by (recall (3.1))

$$
f(x, s)=\lambda(x, s) Q_{d-s}(x-c), g_{i}(x, s)=f(x, s), g_{j}(x, s)=0 \quad \text { if } j \in\{1,2,3\} \text { and } j \neq i .
$$

We have

$$
\begin{aligned}
|f(x, t)| & \leqq C\left(|x-c|+(d-t)^{1 / 2}\right)^{-3}, \\
|D f(x, t)| & \leqq C\left(|x-c|+(d-t)^{1 / 2}\right)^{-4},
\end{aligned}
$$

and hence the method of the proof of Lemma 5.7 of [1] yields

$$
|(D f * \Psi * \Psi)(x, t)| \leqq C\left(|x-c|+(d-t)^{1 / 2}\right)^{-4} .
$$

From $\varepsilon<\tau / 64$ we obtain

$$
(D f * \Psi * \Psi)(x, t)=0 \quad \text { if } x \notin B(c, \tau / 8) .
$$

If $d-h^{2} \leqq t<d$ then the above, $|c-a|<\tau$, and Lemmas 5.13, 5.6 of [1] yield

$$
\begin{aligned}
& \left|\int_{R^{3}} D_{t} z_{i}(x, t) f(x, t)+z_{i}(x, t) D_{t} f(x, t) d x\right| \\
& \quad=\mid \int_{R^{3}} u_{j}(x, t) z_{i}(x, t)\left(D_{j} f * \Psi * \Psi\right)(x, t) d x
\end{aligned}
$$




$$
\begin{aligned}
& \quad-\int_{R^{3}} z_{j}(x, t) u_{i}(x, t)\left(D_{j} f * \Psi * \Psi\right)(x, t) d x \\
& \quad+\int_{R^{3}} z_{i}(x, t)\left(\Delta f+D_{t} f\right)(x, t) d x \\
& \quad+\int_{R^{3}}\left[\left(w_{k}(t) *(\Delta(\Psi * \Omega * \Omega)-\Delta \Psi)\right)(x)\right][\operatorname{curl}(g)(x, t)]_{k} d x \mid \\
& \subseteq C\left(\int_{B(c, \tau / 8)}|u(x, t)||z(x, t)|\left(|x-c|+(d-t)^{1 / 2}\right)^{-4} d x\right) \\
& \quad+\int_{B(a, 5 \tau / 4)}|z(x, t)|\left|\left(\Delta f+D_{t} f\right)(x, t)\right| d x \\
& +C\left\|w^{0}\right\|_{2}\|\Delta(\Psi * \Omega * \Omega)-\Delta \Psi\|_{2}\left(\int_{B(a, 5 \tau / 4)}|D g(x, t)| d x\right) .
\end{aligned}
$$

Since $h<\tau\left[\right.$ see (5.3)] and $\left(\Delta f+D_{t} f\right)(x, t)=0$ if the two conditions $|x-c| \leqq \tau / 32$, $d-h^{2} / 2 \leqq t<d$ are satisfied, we conclude $\left\|\Delta f+D_{t} f\right\|_{2} \leqq C h^{-5 / 2}$. Using this, (5.14), (3.26), (5.11), (5.12), $q \geqq p$, and (5.13) we conclude

$$
\begin{aligned}
\left|z_{i}(c, d)\right|= & \left|\lim _{s \rightarrow d^{-}} \int_{R^{3}} z_{i}(x, s) f(x, s) d x\right| \\
= & \left|\lim _{s \rightarrow d^{-}} \int_{d-h^{2}}^{s} \int_{R^{3}} D_{t} z_{i}(x, t) f(x, t)+z_{i}(x, t) D_{t} f(x, t) d x d t\right| \\
\leqq & \left.C \int_{d-h^{2}}^{d} \int_{B(c, \tau / 8)}|u(x, t)||z(x, t)|\left(|x-c|+(d-t)^{1 / 2}\right)^{-4} d x d t\right) \\
& +\left(\int_{d-h^{2}}^{d} \int_{B(a, 5 \tau / 4)}|z(x, t)|^{2} d x d t\right)^{1 / 2}\left\|\Delta f+D_{t} f\right\|_{2} \\
& +C\left\|w^{0}\right\|_{2}\left(\int_{d-h^{2}}^{d} \int_{B(a, 5 \tau / 4)}|D g(x, t)| d x d t\right) \varepsilon \\
\leqq & C \tau^{1 / 3} 2^{-q / 3} M(|z|, D(d) \cap G(a, b, \tau, q+2))\left(\int_{b-\tau^{2}}^{b} M(t)^{3} d t\right)^{1 / 3} \\
& +\sum_{k=p+2}^{q+1} C \tau^{-3} 2^{3 k} I\left(|D u|^{2}, T\left(a, b, \tau, \tau^{2}, \tau 2^{-k+2}\right)\right) \\
& +C \tau^{-4 / 3} 2^{2 q}\left(\int_{b-\tau^{2}}^{b} M(t)^{3} d t\right)^{2 / 3} \\
& +C \tau^{-3} 2^{3 p}\left(\int_{d-h^{2}}^{d} B(a, 5 \tau / 4)\right. \\
& +C\left(\int_{d-h^{2}}^{d} \int_{B(a, 5 \tau / 4)}|D u(x, t)|^{2} d x d t\right)^{1 / 2} h^{-5 / 2}+C\left\|w^{0}\right\|_{2} h \varepsilon .
\end{aligned}
$$

The conclusion of the lemma follows from the above, (5.3), and $q \geqq p$.

\section{An Estimate for the Vorticity of Approximate Solutions}

The assumptions of Sect. 3 are still in force. 
Lemma 6.1. There exist absolute constants $C_{8}, C_{9}$ with the following property: Suppose $\tau>0, \quad a \in R^{3}, \quad b \in R, \quad B(a, 5 \tau / 4) \subset U, \quad b>\tau^{2}, \quad \varepsilon<\tau / 64, \quad$ and $M(t)$ $=\max \{|u(x, t)|: x \in B(a, 5 \tau / 4)\}$. Suppose also

Then (recall (5.1))

$$
\begin{array}{cc}
\int_{b-\tau^{2}}^{b} M(t)^{3} d t \leqq C_{8} \tau^{-1}, & \\
I\left(|D u|^{2}, T\left(a, b, \tau, \tau^{2}, r\right)\right) \leqq r & \text { if } 0<r \leqq \tau, \\
I\left(|D u|^{2}, K\left(a, b-\tau^{2}+s, 5 \tau / 4, s\right)\right) \leqq \tau^{-1} S & \text { if } 0<s \leqq \tau^{2}, \\
\left\|w^{0}\right\|_{2} \leqq \tau^{-3} \varepsilon^{-1} &
\end{array}
$$

$$
|z(x, t)| \leqq 4 C_{9} \tau^{-2} \quad \text { if }(x, t) \in K\left(a, b, \tau / 2,3 \tau^{2} / 4\right) .
$$

Proof. Choose $C_{8}>0$ so that $2^{4} C_{7} C_{8}^{1 / 3} \leqq 1 / 4$. Then choose $C_{9}>0$ so that $2^{6} C_{7}$ $+C_{7} C_{8}^{2 / 3}+3 C_{7} \leqq C_{9} / 4$. Once again we use the method of the proof of Lemma 3.1 of [1]. We construct a continuous function

such that

$$
f: \text { interior }\left(K\left(a, b, \tau, \tau^{2}\right)\right) \rightarrow R^{+}
$$

$$
C_{9} 2^{2 i} \tau^{-2} \geqq f(x, t) \geqq C_{9} 2^{2(i-1)} \tau^{-2} \quad \text { if }(x, t) \in G(a, b, \tau, i) \sim G(a, b, \tau, i-1)
$$

for $i=1,2,3, \ldots$. As before, we define $G(a, b, \tau, 0)$ to be the empty set. Again, we have

$$
f(x, t) \leqq C_{9} 2^{2 i} \tau^{-2} \quad \text { if }(x, t) \in G(a, b, \tau, i)
$$

We will prove

$$
|z(x, t)| \leqq f(x, t) \quad \text { for all }(x, t) \in \operatorname{interior}\left(K\left(a, b, \tau, \tau^{2}\right)\right) .
$$

Assume that (6.8) is false. Then we can find $(c, d) \in \operatorname{interior}\left(K\left(a, b, \tau, \tau^{2}\right)\right)$ such that

$$
\begin{gathered}
|z(c, d)|=f(c, d), \\
|z(x, t)| \leqq f(x, t) \quad \text { if }(x, t) \in D(d) \cap \text { interior }\left(K\left(a, b, \tau, \tau^{2}\right)\right) .
\end{gathered}
$$

Using this $(c, d)$ we define the integers $p, n, q$ by means of (2.2) and $q$ $=\max \{n, p\}$. Since $(c, d) \notin$ interior $(G(a, b, \tau, q)),(6.6)$ and (6.9) yield

$$
|z(c, d)|=f(c, d) \geqq C_{9} 2^{2 q} \tau^{-2} .
$$

Using (6.3) and the definition of $p$ we find

$$
\begin{aligned}
& \int_{d-\tau^{2} 2}^{d} \int_{-2(p+2)}|D u(x, t)|^{2} d x d t \\
& \quad \leqq \int_{b-\tau^{2}}^{d} \int_{B(a, 5 \tau / 4)}|D u(x, t)|^{2} d x d t \leqq \tau^{-1}\left(d-\left(b-\tau^{2}\right)\right) \leqq \tau 2^{-2 p}
\end{aligned}
$$


Using (6.11), Lemma 5.1, (6.10), (6.7), (6.1), (6.2), $q \geqq p \geqq 0,(6.1),(6.12)$, and (6.4) we obtain

$$
\begin{aligned}
& \tau^{-2} 2^{2 q} C_{9} \leqq|z(c, d)| \\
& \leqq C_{7} \tau^{1 / 3} C_{9} 2^{2(q+2)} \tau^{-2} C_{8}^{1 / 3} \tau^{-1 / 3} \\
&+\sum_{k=p+2}^{q+1} C_{7} \tau^{-3} 2^{3 k} 2^{-k+2}+C_{7} \tau^{-4 / 3} 2^{2 q}\left(C_{8} \tau^{-1}\right)^{2 / 3} \\
&+C_{7} \tau^{-3} 2^{3 p}\left(\tau 2^{-2 p}\right)+C_{7} \tau^{-5 / 2} 2^{5 p / 2}\left(\tau 2^{-2 p}\right)^{1 / 2}+C_{7} \tau^{-3} \tau \\
& \leqq \tau^{-2} 2^{2 q}\left(2^{4} C_{7} C_{9} C_{8}^{1 / 3}\right)+\tau^{-2} 2^{2 q}\left(2^{6} C_{7}\right)+\tau^{-2} 2^{2 q}\left(C_{7} C_{8}^{2 / 3}\right)+\tau^{-2} 2^{p} C_{7} \\
&+\tau^{-2} 2^{3 p / 2} C_{7}+\tau^{-2} C_{7} \\
& \leqq(1 / 4) \tau^{-2} 2^{2 q} C_{9}+\tau^{-2} 2^{2 q}\left(2^{6} C_{7}+C_{7} C_{8}^{2 / 3}+C_{7}+C_{7}+C_{7}\right) \\
& \leqq(1 / 4) \tau^{-2} 2^{2 q} C_{9}+(1 / 4) \tau^{-2} 2^{2 q} C_{9}=(1 / 2) \tau^{-2} 2^{2 q} C_{9} .
\end{aligned}
$$

This is a contradiction. Hence (6.8) must be true. Setting $i=1$ in (6.7) yields (6.5).

Lemma 6.2. There exist absolute constants $C_{10}, C_{11}$ with the following property: Suppose $\sigma>0, a \in R^{3}, b \in R, B(a, 5 \sigma / 4) \subset U, b>\sigma^{2}$, and $\varepsilon<\sigma / 128$. Suppose also

$$
\begin{gathered}
\int_{b-\sigma^{2}}^{b}(\max \{|u(x, t)|: x \in B(a, 5 \sigma / 4)\})^{3} d t \leqq C_{10} \sigma^{-1}, \\
I\left(|D u|^{2}, K\left(a, b, 2 \sigma, \sigma^{2}\right)\right) \leqq C_{10} \sigma \\
\left\|w^{0}\right\|_{2} \leqq \sigma^{-3} \varepsilon^{-1}
\end{gathered}
$$

Then

$$
|z(x, t)| \leqq C_{11} \sigma^{-2} \quad \text { if }(x, t) \in K\left(a, b, \sigma / 4,3 \sigma^{2} / 16\right)
$$

Proof. As in the proof of Lemma 4.2, we find $C_{10} \leqq C_{8}$ such that (6.14) implies that (6.2), (6.3) hold for some $\tau$ such that $\sigma / 2<\tau<\sigma$. Then (6.1) is a consequence of (6.13) and (6.4) is a consequence of (6.15). Using Lemma 6.1 we conclude (6.16).

Lemma 6.3. There exist absolute constants $\eta<1, C_{12}, C_{13}$ with the following property: Suppose $\sigma>0, a \in R^{3}, b \in R, \varepsilon<\eta \sigma / 32, B(a, 5 \sigma / 4) \subset U$, and $b>\sigma^{2}$. Suppose also that

$$
\begin{gathered}
I\left(|D u|^{2}, K\left(a, b, 3 \sigma, \sigma^{2}\right)\right) \leqq C_{12} \sigma, \\
I\left(|u|^{3}, K\left(a, b, 4 \sigma, \sigma^{2}\right)\right) \leqq C_{12} \sigma^{2}, \\
\left\|w^{0}\right\|_{2} \leqq C_{12} \sigma^{-3} \varepsilon^{-1} .
\end{gathered}
$$

Then

$$
|z(x, t)| \leqq C_{13}(\eta \sigma)^{-2} \quad \text { if }(x, t) \in K\left(a, b, \eta \sigma,(\eta \sigma)^{2}\right) .
$$

Proof. Let $\eta>0$ be small enough so that $(4 \eta)^{2} \leqq 3 / 16,5 \eta \leqq 1 / 4, C_{6} \leqq C_{10}(4 \eta)^{-1}$. Then choose $C_{12}>0$ small enough so that $C_{12} \leqq C_{10}(4 \eta), \quad C_{12} \leqq C_{5}$, 
$C_{12} \leqq(4 \eta)^{-3}$. Then Lemma 4.2 yields

$$
\int_{b-(4 \eta \sigma)^{2}}^{b}(\max \{|u(x, t)|: x \in B(a, 5(4 \eta \sigma) / 4)\})^{3} d t \leqq C_{6} \sigma^{-1} \leqq C_{10}(4 \eta \sigma)^{-1}
$$

We also have

$$
\begin{gathered}
I\left(|D u|^{2}, K\left(a, b, 2(4 \eta \sigma),(4 \eta \sigma)^{2}\right)\right) \leqq I\left(|D u|^{2}, K\left(a, b, 2 \sigma, \sigma^{2}\right)\right) \leqq C_{12} \sigma \leqq C_{10}(4 \eta \sigma) \\
\left\|w^{0}\right\|_{2} \leqq C_{12} \sigma^{-3} \varepsilon^{-1} \leqq(4 \eta \sigma)^{-3} \varepsilon^{-1}
\end{gathered}
$$

Lemma 6.2 with $\sigma$ replaced by $4 \eta \sigma$ yields $|z(x, t)| \leqq C_{11}(4 \eta \sigma)^{-2}$ if $(x, t) \in K\left(a, b, \eta \sigma,(\eta \sigma)^{2}\right)$.

Lemma 6.4. There exist an absolute constant $C_{14}$ and a number $N$, where $N$ depends only on $\left\|w^{0}\right\|_{2}$, such that the following is true. Suppose $0<\tau<1$,

and

$$
\varepsilon<\eta(4 \tau / 5) / 32, \quad\left\|w^{0}\right\|_{2} \leqq C_{12}(4 \tau / 5)^{-3} \varepsilon^{-1},
$$

$$
T=\left\{(x, t) \in R^{3} \times R: t \geqq \tau^{2} \text { and distance }\left(x, R^{3} \sim U\right) \geqq \tau\right\} .
$$

Then there exist $\left(x_{1}, t_{1}\right),\left(x_{2}, t_{2}\right), \ldots,\left(x_{M}, t_{M}\right)$ where $\left(x_{j}, t_{j}\right) \in T, M \leqq N \tau^{-5 / 3}$, and

$$
|(\operatorname{curl}(u))(x, t)| \leqq C_{14} \tau^{-2} \quad \text { if }(x, t) \in T \sim \bigcup_{j=1}^{M} K\left(x_{j}, t_{j}, \tau, \tau^{2}\right) .
$$

Proof. We define $\sigma=4 \tau / 5$. Observe that there exist $\left(a_{1}, b_{1}\right),\left(a_{2}, b_{2}\right), \ldots$ and an absolute constant $C_{15}$ such that $\left(a_{i}, b_{i}\right) \in R^{3} \times R, B\left(a_{i}, \tau\right) \subset U, b_{i}>\tau^{2}$,

$$
T \subset \bigcup_{i=1}^{\infty} K\left(a_{i}, b_{i}, \eta \sigma,(\eta \sigma)^{2}\right)
$$

and for every $(x, t) \in R^{3} \times R$ there exist at most $C_{15}$ integers $i$ such that $(x, t) \in K\left(a_{i}, b_{i}, 4 \sigma, \sigma^{2}\right)$. Hölder's inequality yields

$$
I\left(|u|^{3}, K\left(a_{i}, b_{i}, 4 \sigma, \sigma^{2}\right)\right) \leqq C_{16} \sigma^{1 / 2}\left(I\left(|u|^{10 / 3}, K\left(a_{i}, b_{i}, 4 \sigma, \sigma^{2}\right)\right)\right)^{9 / 10} .
$$

From Lemma 5.6, Definition 5.10, Definition 5.14, and Lemma 5.17 of [1] we conclude

$$
\begin{aligned}
& \int_{0}^{\infty} \int_{R^{3}}|D u(x, t)|^{2} d x d t \leqq(1 / 2)\left\|w^{0}\right\|_{2}^{2}, \\
& \int_{0}^{\infty} \int_{R^{3}}|u(x, t)|^{10 / 3} d x d t \leqq C\left\|w^{0}\right\|_{2}^{10 / 3} .
\end{aligned}
$$

Hence the number of integers $i$ for which the inequality

$$
I\left(|D u|^{2}, K\left(a_{i}, b_{i}, 3 \sigma, \sigma^{2}\right)\right) \leqq C_{12} \sigma
$$

fails to hold is at most $C\left\|w^{0}\right\|_{2}^{2} \sigma^{-1}$. Similarly, the number of integers $i$ for which the inequality

$$
I\left(|u|^{10 / 3}, K\left(a_{i}, b_{i}, 4 \sigma, \sigma^{2}\right)\right) \leqq\left(C_{12} C_{16}^{-1}\right)^{10 / 9} \sigma^{5 / 3}
$$


fails to hold is at most $C\left\|w^{0}\right\|_{2}^{10 / 3} \sigma^{-5 / 3}$. Combining this with (6.22) we find that the number of integers $i$ or which the inequality

$$
I\left(|u|^{3}, K\left(a_{i}, b_{i}, 4 \sigma, \sigma^{2}\right)\right) \leqq C_{12} \sigma^{2}
$$

fails to hold is at most $C\left\|w^{0}\right\|_{2}^{10 / 3} \sigma^{-5 / 3}$. Since $\tau<1$ we conclude that there exist integers $i_{1}, i_{2}, \ldots, i_{M}$ such that

$$
\begin{gathered}
M \leqq N \tau^{-5 / 3} \text { where } N \text { depends only on }\left\|w^{0}\right\|_{2}, \\
I\left(|D u|^{2}, K\left(a_{i}, b_{i}, 3 \sigma, \sigma^{2}\right)\right) \leqq C_{12} \sigma \quad \text { if } i \notin\left\{i_{1}, \ldots, i_{M}\right\}, \\
I\left(|u|^{3}, K\left(a_{i}, b_{i}, 4 \sigma, \sigma^{2}\right)\right) \leqq C_{12} \sigma^{2} \quad \text { if } i \notin\left\{i_{1}, \ldots, i_{M}\right\} .
\end{gathered}
$$

From Lemma 6.3 we conclude that $|z(x, t)| \leqq C_{13}(\eta \sigma)^{-2}$ if $(x, t) \in K\left(a_{i}, b_{i}, \eta \sigma,(\eta \sigma)^{2}\right)$ and $i \notin\left\{i_{1}, \ldots, i_{M}\right\}$. Now (6.21) implies that $|z(x, t)|$ is bounded by $C_{13}(\eta \sigma)^{-2}$ on the set

$$
T \sim \bigcup_{j=1}^{M} K\left(a_{i_{j}}, b_{i_{j}}, \eta \sigma,(\eta \sigma)^{2}\right) .
$$

The conclusion follows from $\eta<1$ and (5.1).

The theorem in Sect. 1 now follows easily. The function $u$ was constructed using fixed $0<\alpha<\varepsilon$. In Sect. 6 of [1] it is shown that a sequence of such functions $u$ with $\varepsilon \rightarrow 0$ converges weakly to a weak solution to the NavierStokes equations with initial condition $w^{0}$ and the adherence condition at the boundary of $U$. The conclusion follows from Lemma 6.4 and a compactness argument.

\section{References}

1. Scheffer, V.: Commun. Math. Phys. 73, 1-42 (1980)

Communicated by J. Glimm

Received December 24, 1980 